Article

\title{
Ameliorated Electrical-Tree Resistant Characteristics of UV-Initiated Cross-Linked Polyethylene Nanocomposites with Surface-Functionalized Nanosilica
}

\author{
Yong-Qi Zhang ${ }^{1}$, Ping-Lan $\mathrm{Yu}^{2}$, Wei-Feng Sun ${ }^{1, *}$ and Xuan Wang ${ }^{1, *}$ \\ 1 Key Laboratory of Engineering Dielectrics and Its Application, Ministry of Education, School of Electrical and \\ Electronic Engineering, Harbin University of Science and Technology, Harbin 150080, China; \\ jonegen@126.com \\ 2 Chaozhou Power Supply Bureau, Guangdong Power Grid Co. Ltd., Chaozhou 521000, China; \\ kingstel@163.com \\ * Correspondence: sunweifeng@hrbust.edu.cn (W.-F.S.); topix_xuan@sina.com (X.W.); Tel.: +86-158-4659-2798 (W.-F.S.)
}

check for updates

Citation: Zhang, Y.-Q.; Yu, P.-L.; Sun, W.-F.; Wang, X. Ameliorated Electrical-Tree Resistant Characteristics of UV-Initiated Cross-Linked Polyethylene Nanocomposites with Surface-Functionalized Nanosilica. Processes 2021, 9, 313. https:// doi.org/10.3390/pr9020313

Academic Editors: Shaghayegh Hamzehlou and M. Ali Aboudzadeh Received: 23 January 2021

Accepted: 5 February 2021

Published: 8 February 2021

Publisher's Note: MDPI stays neutral with regard to jurisdictional claims in published maps and institutional affiliations.

Copyright: (c) 2021 by the authors. Licensee MDPI, Basel, Switzerland. This article is an open access article distributed under the terms and conditions of the Creative Commons Attribution (CC BY) license (https:// creativecommons.org/licenses/by/ $4.0 /)$.

\begin{abstract}
Given the high interest in promoting crosslinking efficiency of ultraviolet-initiated crosslinking technique and ameliorating electrical resistance of crosslinked polyethylene (XLPE) materials, we have developed the funcionalized-SiO 2 /XLPE nanocomposites by chemically grafting auxiliary crosslinkers onto nanosilica surfaces. Trimethylolpropane triacrylate (TMPTA) as an effective auxiliary crosslinker for polyethylene is grafted successfully on nanosilica surfaces through thioleneclick chemical reactions with coupling agents of sulfur silanes and 3-mercaptopropyl trimethoxy silane (MPTMS), as characterized by nuclear magnetic resonance and Fourier transform infrared spectroscopy. The functionalized $\mathrm{SiO}_{2}$ nanoparticles could be dispersively filled into polyethylene matrix even at a high filling content that would generally produce agglomerations of neat $\mathrm{SiO}_{2}$ nanofillers. Ultraviolet-initiated polyethylene crosslinking reactions are efficiently stimulated by TMPTA grafted onto surfaces of $\mathrm{SiO}_{2}$ nanofillers, averting thermal migrations out of polyethylene matrix. Electrical-tree pathways and growth mechanism are specifically investigated by elucidating the microscopic tree-morphology with fractal dimension and simulating electric field distributions with finite-element method. Near nano-interfaces where the shielded-out electric fluxlines concentrate, the highly enhanced electric fields will stimulate partial discharging and thus lead to the electrical-trees being able to propagate along the routes between nanofillers. Surface-modified $\mathrm{SiO}_{2}$ nanofillers evidently elongate the circuitous routes of electrical-tree growth to be restricted from directly developing toward ground electrode, which accounts for the larger fractal dimension and shorter length of electrical-trees in the functionlized-SiO ${ }_{2} / \mathrm{XLPE}$ nanocomposite compared with XLPE and neat-SiO $\mathrm{S}_{2}$ /XLPE nanocomposite. Polar-groups on the modified nanosilica surfaces inhibit electrical-tree growth and simultaneously introduce deep traps impeding charge injections, accounting for the significant improvements of electrical-tree resistance and dielectric breakdown strength. Combining surface functionalization and nanodielectric technology, we propose a strategy to develop XLPE materials with high electrical resistance.
\end{abstract}

Keywords: nanodielectrics; crosslinked polyethylene; auxiliary crosslinker; electrical tree; dielectric breakdown strength

\section{Introduction}

Due to the prospective advantages of high production rate, low raw material cost, and minimal radiation damage, ultraviolet (UV) crosslinking technology has attracted considerable focus in present years [1-3]. Related researches for UV-initiated crosslinking polyethylene (UV-XLPE) are now primarily focused on optimizing UV irradiation light sources and developing photon-initiation systems to improve electrical insulation performances $[4,5]$. In order to gain high photon-initiation yields in crosslinking process, 
effective photon-initiators are capable of efficiently absorbing UV irradiation at a specific wavelength range consistent with UV irradiation spectra. The photon-initiation system consisting of benzophenone (BP) and trimethylolpropane triacrylate (TMPTA) has be verified to efficiently expedite polyethylene crosslinking reactions for the industrial manufacture of high-voltage insulation cables [6]. However, given the low molecular weights and poor compatibility with polyethylene molecules, photon-initiator and auxiliary-crosslinker molecules can easily diffuse out of polymeric blends even at ambient temperature, leading to reductions of UV-initiation efficiency and XLPE crosslinking degree [7]. Meanwhile, photon-initiation systems of small molecules with high volatility are liable to be evaporated and deposited on a UV-source lampshade at an elevated temperature in UV-initiated crosslinking process, which will corrode irradiation instruments and abate UV-light transmission efficiency [8]. It is thereby urgent to develop a novel photon-initiation system or scheme with high polyethylene-compatibility and low thermal volatility for UV-initiation technology in the manufacture of XLPE high-voltage cables.

Inorganic nanoparticles incorporated into polymer matrix (polymer dielectric nanocomposites, nanodielectrics) can comprehensively present amelioration in space charge characteristics, electrical breakdown field, electrical-tree resistance, thermal conductivity, thermostability, and mechanical properties $[9,10]$. Accordingly, the surface modification technology of nanodielectrics provides a strategy for further improving the electrical, thermal, and mechanical properties of cable insulating materials [11]. Surface-modifications ensure a high dispersion of nanofillers maintaining a small size in polymer matrix, which can highly participate in dynamic exchange reactions, thus leading to improved mechanical properties of nanocomposites [12]. By modifying nano-capsules in geometry and size to adjust their interaction with electric field, the insulation defects caused by dielectric breakdown or electrical-trees can be well repaired, which has been widely used in self-healing insulating materials in recent years [13]. Attributed to the extensive source, large specific surface area, high reactivity, non-toxic and pollution-free chemistry, and visible light transparency, $\mathrm{SiO}_{2}$ nanoparticles have been comprehensively utilized for developing UV-cured nanocomposites such as $\mathrm{SiO}_{2}$ /acrylic polyurethane, $\mathrm{SiO}_{2}$ /epoxy resin, and $\mathrm{SiO}_{2} /$ methacrylate to improve the mechanical and electrical properties of polymers [14-16].

In the present paper, the migration and volatilization of small molecular photoncrosslinking system are circumvented by utilizing $\mathrm{SiO}_{2}$ nanoparticles with the surfaces being grafted by auxiliary crosslinker in combination with a large molecular photoninitiator to improve crosslinking degree and insulation performances. Conforming to thiolene-click chemistry and nano-surface modification $[17,18]$, the auxiliary crosslinker TMPTA is chemically grafted onto nanosilica surfaces to acquire an auxiliary crosslinking function by using 3-mercaptopropyl trimethoxy silane (MPTMS). The dispersion of $\mathrm{SiO}_{2}$ nanofillers in polyethylene matrix could thereby be improved due to the well compatibility of TMPTA with polyethylene molecules. Accordingly, functionalized-SiO $\mathrm{S}_{2} / \mathrm{XLPE}$ nanocomposites have been developed in a combination of UV-initiation, chemical surface modification, and nanodielectrics. After molecular and micro-structure characterizations of nuclear magnetic resonance, infrared spectroscopy, and scanning electron microscopy, the electrical-tree resistance and insulation performance of functionalized-SiO ${ }_{2} / \mathrm{XLPE}$ nanocomposites are investigated together with finite-element electric field simulations and fractal dimension analyses to elucidate the mechanism of improving electrical resistance.

\section{Experimental Schemes}

\subsection{Material Preparations}

Pristine materials of preparing nanocomposites are used as follows: linear low density polyethylene (LLDPE, DFDA-7042, Petrochina Jilin Petrochemical Co. Ltd., Changchun, China) as the matrix material; triallyl isocyanurate (TMPTA, chemically pure, Macklin Biochemical Technology Co. Ltd., Shanghai, China) as the auxiliary crosslinker; 4-Hydroxyl benzophenone laurate (BPL, chemically pure, Harbin University of Science and Technology, Harbin, China) as the photon-initiator; pentaerythritol ester (Irganox1010, Shanyi Plas- 
tics Co. Ltd., Dongguan, China) as the antioxidant; 3-merraptnpropylt rimethnxysilane (MPTMS, chemically pure, Jiangsu Heyuan Chemical Co. Ltd., Nanjing, China) as the silane coupling-agent; dichloromethane (DCM), anhydrous ethanol (EtOH), triethylamine (TEA), and silica $\left(\mathrm{SiO}_{2}\right) 20 \mathrm{~nm}$ nanoparticles (Tianjin Fuyu Fine Chemical Co. Ltd., Tianjin, China) for the nanosilica surface modifications.

The surface modification process of functionalizing $\mathrm{SiO}_{2}$ nanoparticles with an auxiliary crosslinker is schematically illustrated in Figure 1, and implemented as follows: $3.92 \mathrm{~g}$ MPTMS (0.02 mol), $0.23 \mathrm{~g}$ TEA, and $4.98 \mathrm{~g}$ TMPTA (0.02 mol) are dissolved into a $10 \mathrm{~mL}$ solvent of DCM in nitrogen protection and ice-water bath, and then warmed to room temperature, persisting for $24 \mathrm{~h}$ in thermal insulation to finally obtain the liquid product (MTMPTA) with 78\% yield. Furthermore, the dried nanosilica of $10 \mathrm{~g}$ is homogeneously blended into a $100 \mathrm{~mL}$ ethanol solution (3:1) by ultrasonic dispersion treatment for $30 \mathrm{~min}$, after which MTMPTA is instilled under $\mathrm{pH}=4$ with diluted hydrochloric acid. By filtering the suspension and washing with ethanol three times, surface-modified nanosilica (TMPTA-s-SiO ${ }_{2}$ ) is eventually achieved after being hot-degassed for $3 \mathrm{~h}$ at $60^{\circ} \mathrm{C}$ in vacuum.

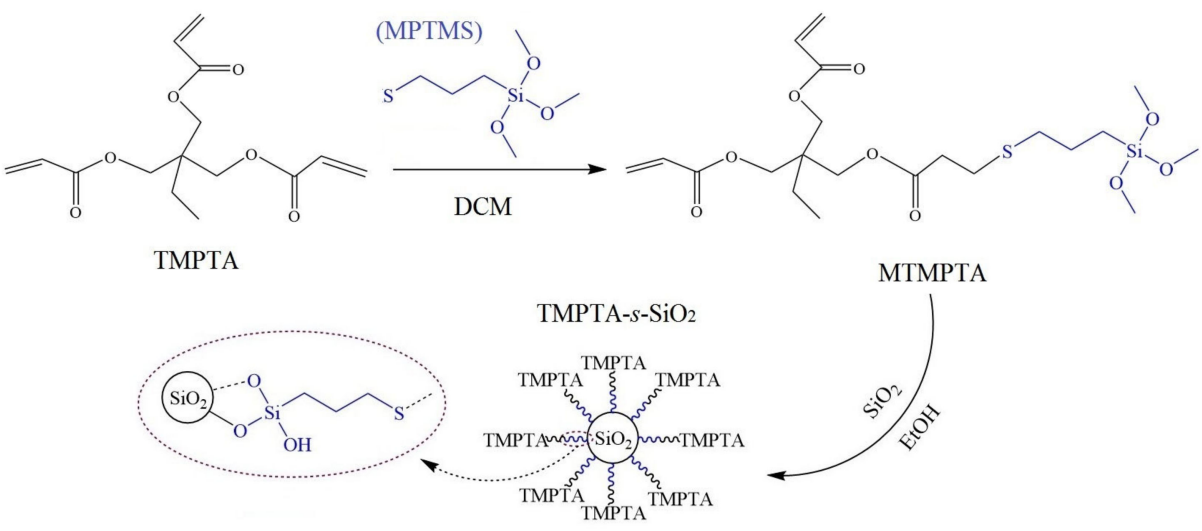

Figure 1. Schematic reactions of trimethylolpropane triacrylate (TMPTA) grafting onto the nanosilica surface.

According to the blending components listed in Table 1, four mixtures for preparing pure and composite XLPE materials are uniformly blended and pressed into film samples at $160{ }^{\circ} \mathrm{C}$ by a plate vulcanizer, in which pressure is increased from 0 to $15 \mathrm{MPa}$ at a rate of $5 \mathrm{MPa} / 5$ min to melt material. For photon-initiated crosslinking reactions, the obtained melting films are irradiated by a light source array of UV LED units (NVSU233A-U365, Riya Electronics Chemistry Co. Ltd., Shanghai, China) for $180 \mathrm{~s}$ under normal temperature and pressure [19], in which the emitting power and light-wavelength are controlled at $1.0 \mathrm{w}$ and $365 \mathrm{~nm}$, respectively, with a light-incidence of a $60^{\circ}$ angle to the film plane [20]. After hot-degassing in short-circuit for $24 \mathrm{~h}$ at $80^{\circ} \mathrm{C}$ in vacuum to eliminate residual chemical by-products and mechanical stresses, pure UV-XLPE and three kinds of UV-XLPE nanocomposites are finally prepared, as listed in Table 1.

Table 1. Blending components (wt $\%$ ) for preparing XLPE nanocomposites.

\begin{tabular}{|c|c|c|c|c|c|c|}
\hline Materials & LLDPE & BPL & TMPTA & TMPTA-s-SiO ${ }_{2}$ & $\mathrm{SiO}_{2}$ & Antioxidant \\
\hline XLPE & 96.7 & 2.0 & 1.0 & 0 & 0 & 0.3 \\
\hline $\begin{array}{c}0.5 \mathrm{wt} \% \text { TMPTA-s- } \\
\mathrm{SiO}_{2} / \text { XLPE }\end{array}$ & 97.2 & 2.0 & 0 & 0.5 & 0 & 0.3 \\
\hline $\begin{array}{c}\text { 1.5wt } \% \text { TMPTA-s- } \\
\mathrm{SiO}_{2} / \text { XLPE }\end{array}$ & 96.7 & 2.0 & 0 & 1.5 & 0 & 0.3 \\
\hline $1.5 \mathrm{wt} \% \mathrm{SiO}_{2} / \mathrm{XLPE}$ & 95.7 & 2.0 & 1.0 & 0 & 1.5 & 0.3 \\
\hline
\end{tabular}

\subsection{Material Characterization and Property Test}

Nuclear magnetic resonance (Bruker- $1,{ }^{1} \mathrm{H}-\mathrm{NMR}$ ) is utilized to determine the hydrogen $(\mathrm{H})$ content according to the type and quantity of $\mathrm{H}$ detected in samples. A Fourier 
transform infrared spectrometer (FTIR-6100, Jiasco Trading Co. Ltd., Shenyang, China) is utilized to characterize molecular structures of TMPTA-s-SiO 2 samples. According to ASTM-D 2675-2011 standard, the solvent-extracted gel contents of XLPE and nanocomposite materials are tested to evaluate the crosslinking degree of XLPE matrix.

Brittlely fractured cross-sections of XLPE nanocomposites are observed by an ultrahigh-resolution scanning electron microscope (SEM, SU8020, Hitachi Co. Ltd., Tokyo, Japan) adjusted to a magnification of $75 \mathrm{k}$ under an acceleration voltage of $5 \sim 10 \mathrm{kV}$. SEM samples need to be sputtered with thin gold layers covering all the fractured surfaces to conduct out the undesirable charges introduced by electron impacts.

For electrical-tree inception and propagation, the sample with an inserted tungsten needle (tip curvature radius $=3 \mu \mathrm{m}$ ) is immersed in silicone oil to avoid creepage discharge, and then a needle electrode is applied with alternating current (AC) high-voltage power at $50 \mathrm{~Hz}$ frequency. The step-up increasing voltage is adopted to reach $2.5 \mathrm{kV}$ at $0.1 \mathrm{kV} / \mathrm{s}$ persisting for $15 \mathrm{~min}$, and then continuously rise up to $0.1 \mathrm{kV} / \mathrm{s}$ until electrical-tree appearing. An optical system consisting of a charge-coupled device (CCD) camera and in situ optical microscope are accessed through the computer interface to observe electrical-tree morphology in time intervals of $5 \mathrm{~min}$. The electrical-tree of $10 \mu \mathrm{m}$ length is specified as identifying tree inception and 10 samples are tested for each material in statistical analyses with 2-parameter Weibull distribution. Electrical-tree propagation is further evaluated though finite-element electrical field simulations under $50 \mathrm{~Hz}$ AC voltage.

Thermally stimulated currents (TSC, Harbin University of Science and Technology, Harbin, China) are tested to evaluate the energy level distributions of charge traps. First, the film samples in $100 \mu \mathrm{m}$ thickness are applied with an electric field of $40 \mathrm{kV} / \mathrm{mm}$ for $30 \mathrm{~min}$ at room temperature. The sample-electrode system in short-circuit is swiftly cooled down to $-30{ }^{\circ} \mathrm{C}$ and stabilized for $10 \mathrm{~min}$, after which TSC through short-circuit samples is consecutively tested with a microcurrent meter when temperature rising from -30 to $175^{\circ} \mathrm{C}$ at a heating rate of $3{ }^{\circ} \mathrm{C} / \mathrm{min}$.

Circular film samples with a diameter and thickness of $80 \mathrm{~mm}$ and $0.1 \mathrm{~mm}$, respectively, are fabricated to measure AC dielectric breakdown strength (DBS) with asymmetric columnar electrodes $(25 \mathrm{~mm}$ and $75 \mathrm{~mm}$ in diameter for high-voltage and ground electrodes, respectively) at the same temperature as electrical-tree experiments. The applied electric field is continuously increased at a rate of $4 \mathrm{kV} / \mathrm{s}$ until attaining the possible maximum voltage that is recorded promptly just before electrical breakdown.

\section{Results and Discussion}

\subsection{Material Characterization}

As indicated from the ${ }^{1} \mathrm{H}-\mathrm{NMR}$ spectrum of MTMPTA molecules in Figure $2 \mathrm{a}$, the successfully synthesized MTMPTA is demonstrated by the peaks at $\delta=5.82,6.38$, and 6.09 ppm identifying the chemical displacements of $\mathrm{H}$ atoms in $-\mathrm{CH}=\mathrm{CH}_{2}$ group, and $\delta=2.74$ and $2.61 \mathrm{ppm}$ indicating the $\mathrm{H}$ atoms on $-\mathrm{CH}_{2}-$ adjacent to sulfur atom (S). FTIR spectra of the functionalized nanoparticles TMPTA-s-SiO 2 grafted with auxiliary crosslinkers are shown in Figure $2 b$. Infrared absorption peaks at $3438 \mathrm{~cm}^{-1}, 2952 \mathrm{~cm}^{-1}$, $2841 \mathrm{~cm}^{-1}$, and $2561 \mathrm{~cm}^{-1}$ indicate the atomic vibrations of $\mathrm{Si}-\mathrm{OH}$ on nanosilica surfaces, methyl, methylene, and -SH in MPTMS molecules, respectively. Characteristic peaks at $1726 \mathrm{~cm}^{-1}$ and $1637 \mathrm{~cm}^{-1}$, respectively, originate from stretching vibrations of doublebonds in $-\mathrm{C}=\mathrm{O}$ and $-\mathrm{CH}=\mathrm{CH}_{2}$ groups of TMPTA molecules. The simultaneously arising peaks of methyl/methylene $\left(2957 \mathrm{~cm}^{-1}\right)$, carbonyl $\left(1717 \mathrm{~cm}^{-1}\right)$, and ethylene $\left(1638 \mathrm{~cm}^{-1}\right)$ in the infrared spectrum of TMPTA-s-SiO ${ }_{2}$ verify that TMPTA has been substantially grafted to nanosilica surfaces. The ${ }^{1} \mathrm{H}-\mathrm{NMR}$ and FTIR spectra imply that the grafting rate of TMPTA on the surfaces of TMPTA-s-SiO ${ }_{2}$ nanoparticles can be adjusted by modifying MTMPTA content in surface modification processes. Nevertheless, the density of TMPTA in TMPTA$s-\mathrm{SiO}_{2} /$ XLPE nanocomposites can also be controlled by increasing the concentration of TMPTA-s-SiO 2 nanofillers to ameliorate the crosslinking degree and electrical resistance. 
Thereby, $30 \mathrm{wt} \%$ dosage of MTMPTA is specialized in the synthesis of TMPTA-s-SiO${ }_{2}$ nanoparticles which are subsequently used to prepare XLPE nanocomposites.

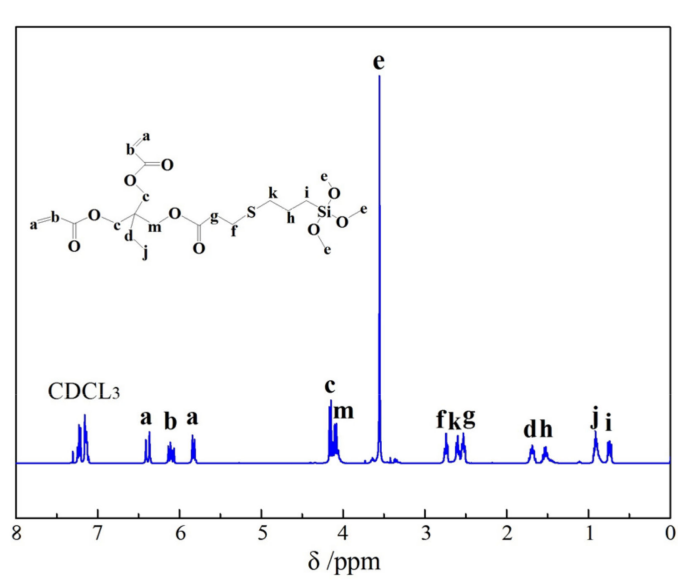

(a)

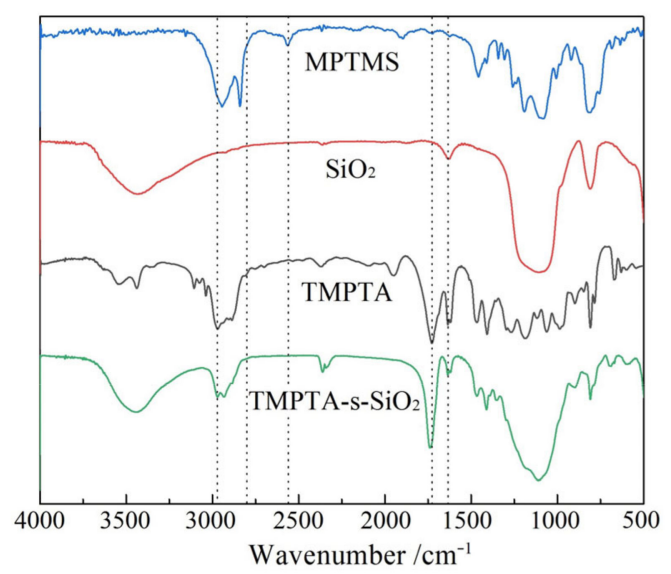

(b)

Figure 2. (a) ${ }^{1} \mathrm{H}-\mathrm{NMR}$ spectrum of MTMPTA molecules and (b) Fourier transform infrared (FTIR) transmission spectra of MPTMS, neat nanosilica, TMPTA, and TMPTA-s-SiO 2 nanoparticles.

\subsection{Crosslinking Degree}

Identical photon-initiator content and irradiation time are applied for polyethylene crosslinking reactions in preparations of UV-initiated XLPE nanocomposites to investigate the effect of grafting TMPTA to nanosilica surface on the crosslinking degree of polyethylene matrix, as illustrated by gel content (a general indicator of crosslinking degree) shown in Figure 3. For the $0.5 \mathrm{wt} \%$ and $1.5 \mathrm{wt} \%$ filling content of TMPTA-s-SiO ${ }_{2}$, crosslinking degrees are remarkably increased by $30 \%$ and $120 \%$, respectively, compared with that of pure XLPE. Furthermore, the XLPE nanocomposite prepared by filling neat $\mathrm{SiO}_{2}$ nanoparticles $\left(\mathrm{SiO}_{2} / \mathrm{XLPE}\right.$ nanocomposite) without TMPTA-functionalized surface shows a low crosslinking degree almost identical to that of pure XLPE. As shown in Table 1, the content of dissociative TMPTA added into polyethylene matrix to assist crosslinking reactions approaches $1.0 \mathrm{wt} \%$ for pure XLPE and $\mathrm{SiO}_{2}$ /XLPE nanocomposite, which is much higher than the actual content of TMPTA grafted onto surfaces of $\mathrm{SiO}_{2}$ nanofillers in TMPTA-s$\mathrm{SiO}_{2} /$ XLPE nanocomposites. Additionally, the surface functionalization of $\mathrm{SiO}_{2}$ nanofillers by grafting TMPTA shows a great validity of fixing TMPTA molecules into XLPE matrix to avoid thermal migrations out of crosslinking reaction system and promote the auxiliary crosslinking efficiency. Moreover, the significantly further increment of crosslinking degree by increasing filling content from $0.5 \mathrm{wt} \%$ to $1.5 \mathrm{wt} \%$ of TMPTA-s-SiO ${ }_{2} / \mathrm{XLPE}$ nanocomposites is also a manifestation of the auxiliary function of TMPTA in crosslinking reactions to improve crosslinking degree, which can be stably introduced into polyethylene crosslinking system through additional nanosurface functionalization in nanodielectric technology.

\subsection{Microscopic Morphology of Nanocomposites}

The outmost TMPTA layer cladding $\mathrm{SiO}_{2}$ nanocore possesses a high compatibility with polyethylene molecular-chain and determine the dispersivity and size of nanofillers in XLPE matrix, which accordingly contributes to the acquired improvements in dielectric properties of composite materials. It is indicated from cross-sectional SEM images illustrated in Figure 4 that the surface-modified nanofillers in TMPTA-s-SiO ${ }_{2} / \mathrm{XLPE}$ nanocomposites are majorly distributed in sizes of $<80 \mathrm{~nm}$ with a favorable high dispersivity. It is impossible to completely prevent nanofillers from agglomerating in polyethylene matrix for the composite materials prepared with melting blend method, as shown in Figure $4 \mathrm{c}$ for $1.5 \mathrm{wt} \% \mathrm{SiO}_{2} / \mathrm{XLPE}$ nanocomposite with some $\mathrm{SiO}_{2}$ fillers larger than $150 \mathrm{~nm}$ in diameter. Nevertheless, the surface-modified silica fillers remain in nanoscales even for $1.5 \mathrm{wt} \%$ filling content, as shown in Figure $4 \mathrm{~b}$, which means they fulfill successful nan- 
odielectrics. Compared with $0.5 \mathrm{wt} \%$ filling content, a higher density of TMPTA-s-SiO nanofillers with an almost identical size is uniformly dispersed in XLPE matrix without any nanoparticle agglomeration.

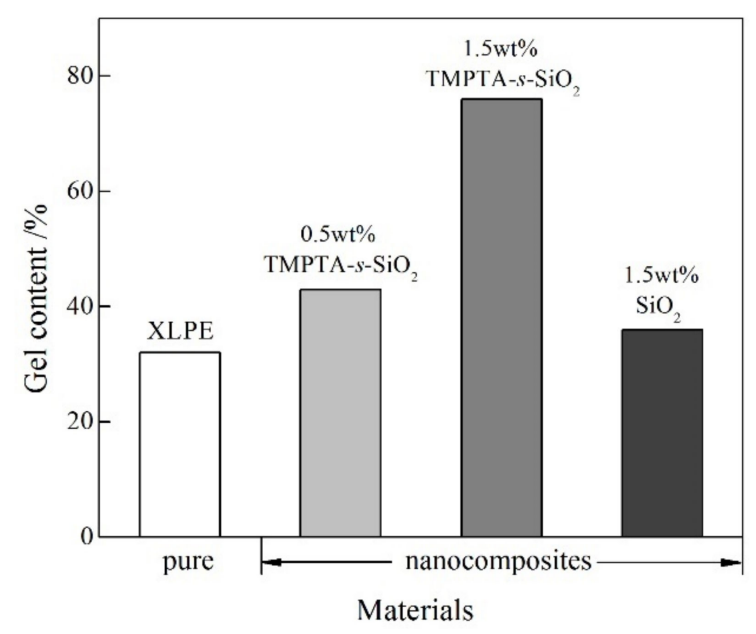

Figure 3. Gel contents of XLPE and its nanocomposites filled with $\mathrm{TMPTA}-\mathrm{s}-\mathrm{SiO}_{2}$ or neat $\mathrm{SiO}_{2}$ nanoparticles.
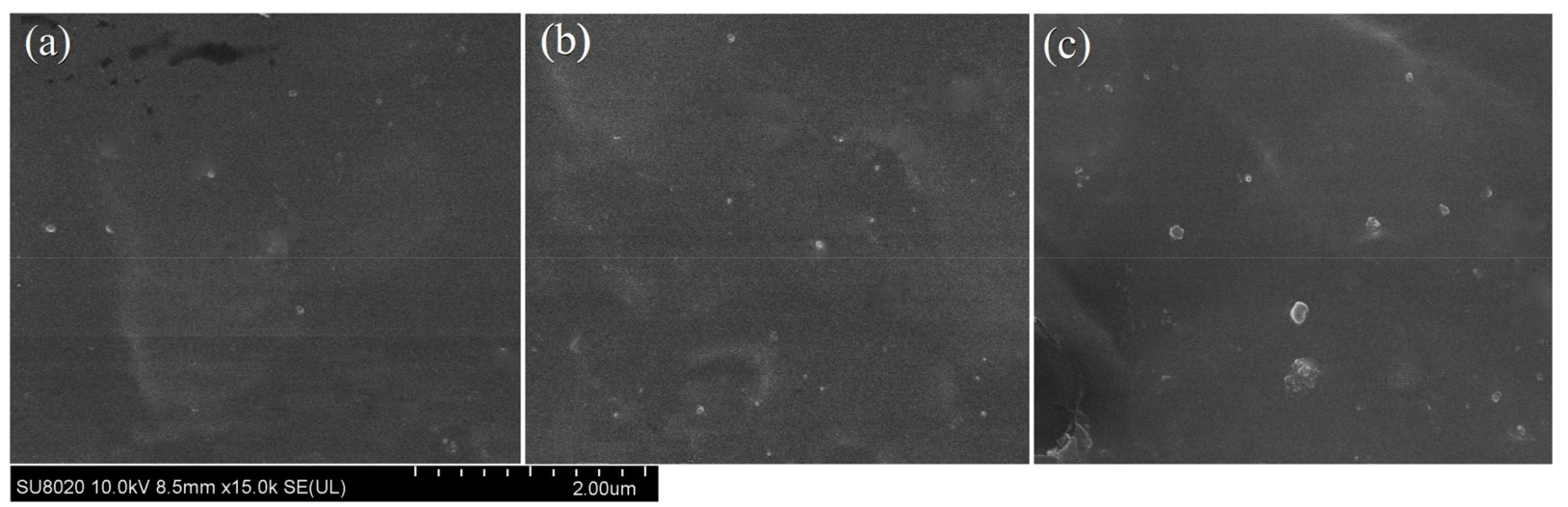

Figure 4. Cross-sectional SEM images: (a) $0.5 \mathrm{wt} \% \mathrm{TMPTA}-\mathrm{s}-\mathrm{SiO}_{2} / \mathrm{XLPE}$, (b) $1.5 \mathrm{wt} \% \mathrm{TMPTA}-\mathrm{s}-\mathrm{SiO}_{2} / \mathrm{XLPE}_{\mathrm{T}}$ and (c) $1.5 \mathrm{wt} \% \mathrm{SiO}_{2} /$ XLPE nanocomposites.

\subsection{Electrical Tree Development}

Electrical-tree inception voltages are fitted by 2-parameter Weibull statistics for XLPE and its nanocomposites, as shown in Figure 5. Characteristic inception voltages and shape parameters are listed in Table 2. Despite the almost same shape parameters for all the materials, the characteristic electrical-tree inception voltages of TMPTA-s-SiO 2 /XLPE nanocomposites with $0.5 \mathrm{wt} \%$ and $1.5 \mathrm{wt} \%$ filling contents have been increased to $6.53 \mathrm{kV}$ and $7.15 \mathrm{kV}$, respectively, which are $9 \%$ and $19 \%$ higher than that of pure XLPE, while $\mathrm{SiO}_{2} /$ XLPE nanocomposite represents an almost identical inception voltage ( $2 \%$ higher) to pure XLPE. Therefore, the evident improvement in electrical tree inception voltage is dominantly attributed to the TMPTA-grafting layer on nanosilica surface. It is suggested that the charge carriers (electrons or holes) injected from needle electrodes cannot be accelerated when transporting through TMPTA-s-SiO 2 nanofillers and will be scattered to form divergent discharging channels in high electric field regions. Accordingly, the carbonized discharging channels (tree like damages) will be inhibited from directly developing to the ground electrode plate, as manifested by the shorter electrical-tree length and higher inception voltage (judged by the same standard of tree length) as shown in Figure 5 and Table 2. 


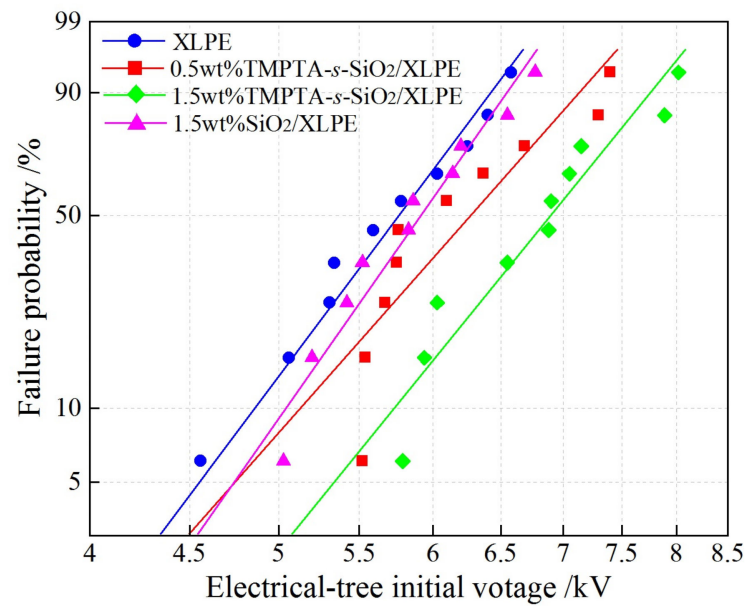

Figure 5. Weibull 2-parameter distributions of the electrical-tree inception voltages for XLPE and its nanocomposites.

Table 2. Characteristic electrical-tree inception voltages at $63.2 \%$ probability and shape parameters of 2-parameter Weibull statistics at 95\% confidence interval.

\begin{tabular}{cccc}
\hline Materials & $\begin{array}{c}\text { Characteristic Inception } \\
\text { Voltage/kV }\end{array}$ & $\begin{array}{c}\text { Shape } \\
\text { Parameter }\end{array}$ & Increment/\% \\
\hline XLPE & 5.99 & 12.37 & \\
$0.5 \mathrm{wt} \% \mathrm{TMPTA}-\mathrm{s}-\mathrm{SiO}_{2} / \mathrm{XLPE}$ & 6.53 & 9.349 & 9 \\
$1.5 \mathrm{wt} \% \mathrm{TMPTA}-\mathrm{s}-\mathrm{SiO}_{2} / \mathrm{XLPE}$ & 7.15 & 10.17 & 19 \\
$1.5 \mathrm{wt} \% \mathrm{SiO}_{2} / \mathrm{XLPE}$ & 6.10 & 11.79 & 2 \\
\hline
\end{tabular}

By applying an AC voltage for $7200 \mathrm{~s}$, after which nearly no change can be observed in tree morphology, the structure features of electrical-trees growing in XLPE and its nanocomposites are pictured by optical microscopy, as shown in Figure 6. TMPTA-s$\mathrm{SiO}_{2} / \mathrm{XLPE}$ nanocomposites present electrical-trees in shapes of disperse, dense, and short shrubbery, while the pure XLPE and $\mathrm{SiO}_{2} / \mathrm{XLPE}$ nanocomposite show pine-branch like electrical-trees in the morphology of sparse branches concentrating around the long main trunks.
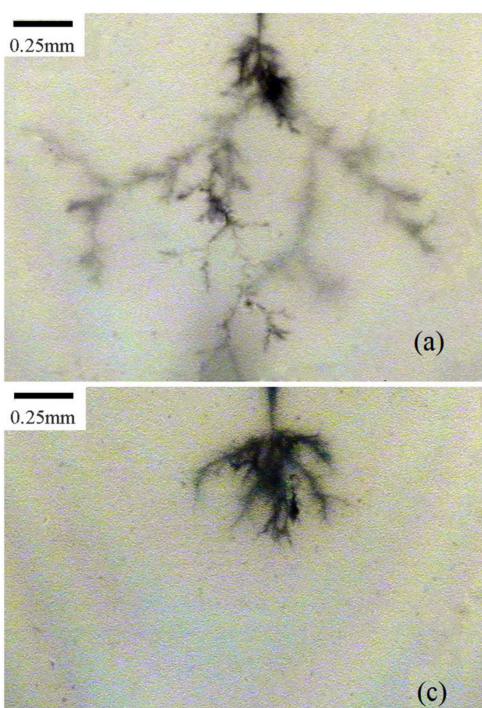
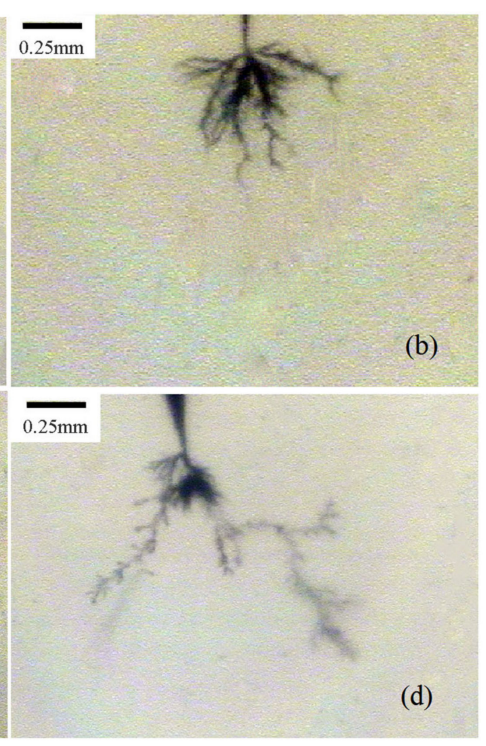

Figure 6. The electrical-tree morphology after $7200 \mathrm{~s}$ in (a) XLPE and the nanocomposites of (b) $0.5 \mathrm{wt} \% \mathrm{TMPTA}-\mathrm{s}-\mathrm{SiO}_{2} / \mathrm{XLPE}$, (c) $1.5 \mathrm{wt} \% \mathrm{TMPTA}-\mathrm{s}-\mathrm{SiO}_{2} / \mathrm{XLPE}$, and (d) $1.5 \mathrm{wt} \% \mathrm{SiO}_{2} / \mathrm{XLPE}$. 
Based on dielectric permittivity and electrical conductivity, the local electric field distributions around needle tip (or considered as the front of electrical-tree) are simulated with finite element method as implemented in COMSOL multi-physics software, with the results being shown in Figure 7. The triangular region in white color represents electrode needle-tip or tree-front. According to the nanofiller sizes observed from SEM images and the molecule dimension of MTMPTA, we model TMPTA-s- $-\mathrm{SiO}_{2}$ and neat $\mathrm{SiO}_{2}$ nanofillers by the smaller shell-core structure and the larger neat sphere respectively, which are dispersively put into the uniform background of XLPE matrix to simulate nanocomposites, as shown in Figure $7 \mathrm{~b}-\mathrm{d}$.
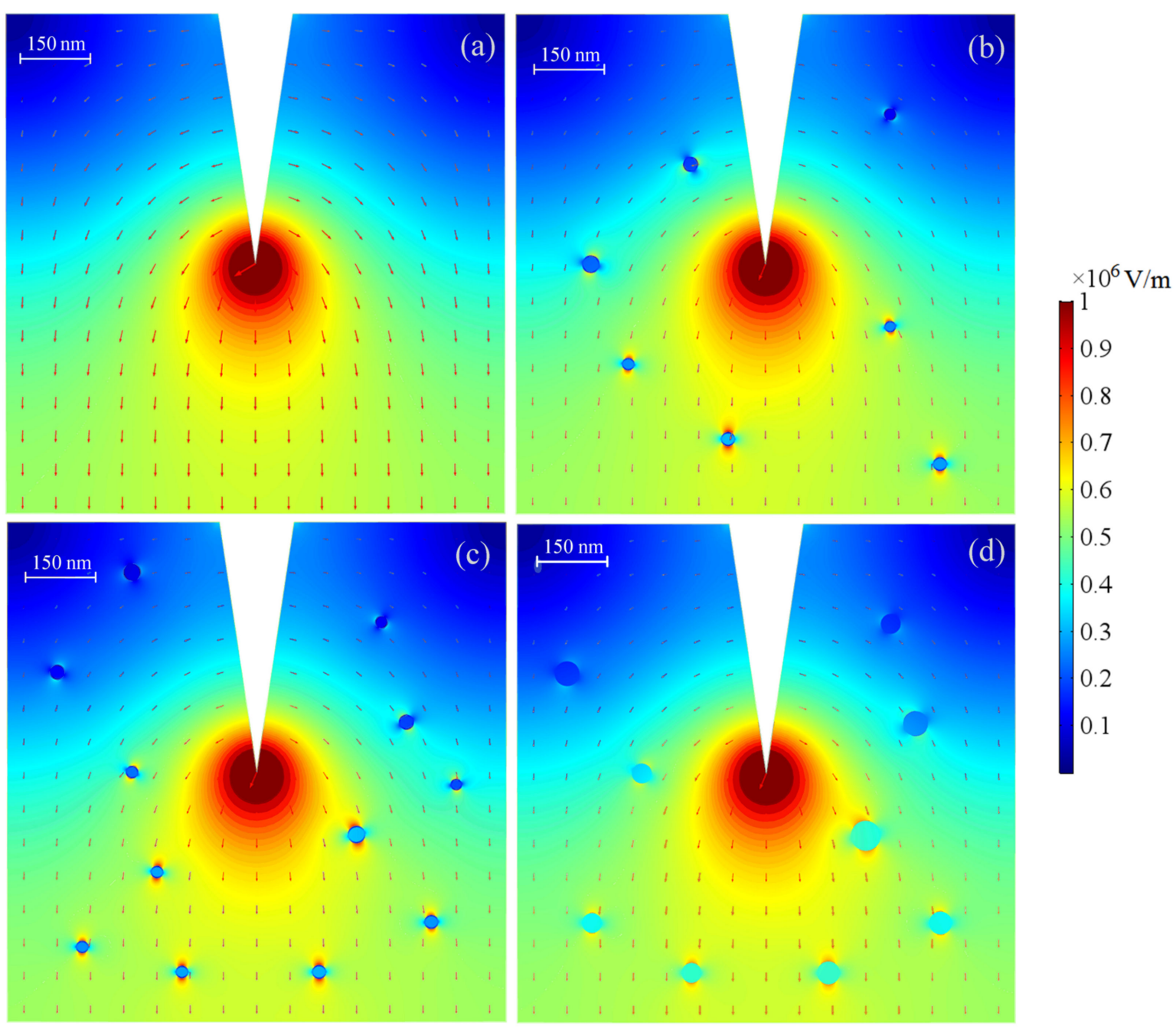

Figure 7. Electric field distributions around initiation tip (identical to tree front) simulated for (a) XLPE, (b) $0.5 \mathrm{wt} \% \mathrm{TMPTA}-$ s-SiO ${ }_{2}$ /XLPE, (c) $1.5 \mathrm{wt} \%$ TMPTA-s-SiO $2 /$ XLPE, and (d) $1.5 \mathrm{wt} \% \mathrm{SiO}_{2} /$ XLPE nanocomposites.

The electric field in $\mathrm{SiO}_{2}$ core of the surface-modified nanofiller is remarkably lower than that of XLPE matrix, leading to an evident concentration of electric fields near two sides of the outer surfaces crossing electric fluxlines between the two adjacent nanofillers as shown in Figure $7 \mathrm{~b}$,c. In contrast, the modeled particles of neat $\mathrm{SiO}_{2}$ nanofillers in XLPE background show unconspicuous discrepancy of the electric fields inside and outside nanosurfaces, as shown in Figure $7 \mathrm{~d}$. The dielectric permittivity of the surface-modified MTMPTA shell is significantly higher than that of $\mathrm{SiO}_{2}$ core and XLPE matrix, so as to expel the electric fluxlines out of nanofillers and accordingly intensify the electric fields (concentrate the continuous electric fluxlines) at the outer surfaces where the electric fluxlines enter and flow out through nanofillers. The much lower and higher electric fields 
in $\mathrm{SiO}_{2}$ core and outside MTMPTA shell respectively than the background electric field of XLPE matrix could be comprehended as "dielectric shielding" and cannot be effectively presented by the neat $\mathrm{SiO}_{2}$ nanofillers with a low dielectric permittivity as XLPE. It is reasonably suggested that the electrical-trees will be apt to form and propagate along the paths between TMPTA-s-SiO 2 nanofillers due to the highly increased spontaneous probability of partial discharge under high electric fields near the surfaces of TMPTA-s$\mathrm{SiO}_{2}$ nanofillers. Consequently, electrical-trees will be more possible to grow consecutively along the network pathways through TMPTA-s $-\mathrm{SiO}_{2}$ nanofillers, which means a larger numbers of circuitous routes for electrical-tree developing from high voltage to ground, as manifested by the higher inception voltage and the larger fractal dimension with shorter tree-length that will be demonstrated in follows.

Fractal structure of electrical-trees can be evaluated from the growth length $L$ along electric field and fractal dimension $D_{\mathrm{f}}$, in which growth quantity $Y$ and tree length $L_{\mathrm{B}}$ as a function of growing time are formularized as follows [21]:

$$
\begin{aligned}
& Y=\left(L / L_{\mathrm{B}}\right)^{D_{\mathrm{f}},} \\
& L=L_{\mathrm{B}}{ }^{\left(1+1 / D_{\mathrm{f}}\right)}\left[\frac{\omega}{2 \pi} \exp \left(\frac{\alpha l \pi \varepsilon E^{2}-U_{0}}{k T}\right)\right]^{1 / D_{\mathrm{f}}} t^{1 / D_{\mathrm{f}}}
\end{aligned}
$$

where $\omega / 2 \pi$ symbolizes the intrinsic frequency of atomic vibrations in polymer materials; $U_{0}$ represents the activation energy of electrical-tree expansions; $\varepsilon$ denotes dielectric permittivity; $E$ signifies local electric field; $\alpha$ indicates thermodynamic parameter of atomic vibrations in polymer materials; $l$ symbolizes the linear dimension of micro-cracks; and $T$ and $k$ are thermodynamic temperature and Boltzmann constant, respectively. Specifically for polyolefin materials such as XLPE, atomic-vibration frequency is predominately located on $\omega / 2 \pi=6.25 \times 10^{12} \mathrm{~Hz}$, and the molecular vibration parameter $\alpha=6.68 \times 10^{-16}$ is determined from activation volume [22]. Meanwhile, the electric field should be calculated approximately as following [22]:

$$
E=\frac{2 U}{r \ln (1+4 R / r)}
$$

where $U=6 \mathrm{kV}$ indicates the electrical voltage applied on needle electrode; $r=3 \mu \mathrm{m}$ identifies the curvature radius of needle tip; and $R=3 \mathrm{~mm}$ denotes the distance from bottom plate to needle tip.

Fractal dimension can quantitatively depict the spatial topological characteristics for characterizing electrical-tree structure. As implemented with MATLAB programming, box counting is performed on the electrical-tree images from optical microscope observations to evaluate the fractal dimension of electrical-trees produced in pure XLPE and XLPE nanocomposites. Tree length versus fractal dimension of XLPE is calculated from Equations (1) and (2) in comparison with experimental results observed from electrical-tree morphology in pure XLPE and $1.5 \mathrm{wt} \% \mathrm{TMPTA}-\mathrm{s}-\mathrm{SiO}_{2} / \mathrm{XLPE}$ nanocomposite, as shown in Figure 8. In macroscopic scale, smaller fractal dimension of electrical-trees means a more tree-development along electric field. For $1.5 \mathrm{wt} \% \mathrm{TMPTA}-\mathrm{s}-\mathrm{SiO}_{2} / \mathrm{XLPE}$ nanocomposite, the absolute variation slope of electrical-tree length vs. fractal dimension is significantly smaller than theoretical values of XLPE (well consistent with experimental results). The electrical-tree development directly towards to ground electrode (tree length) is significantly reduced by the partial discharging routes in the network of high electric fields near the "dielectric shielding" surfaces of TMPTA-s-SiO 2 nanofillers, leading to the expedition of electrical-tree developing along the more circuitous trajectories in dispersive geometries, as indicated by the large fractal dimensions of electrical-trees produced in TMPTA-s-SiO ${ }_{2} /$ XLPE nanocomposites. 


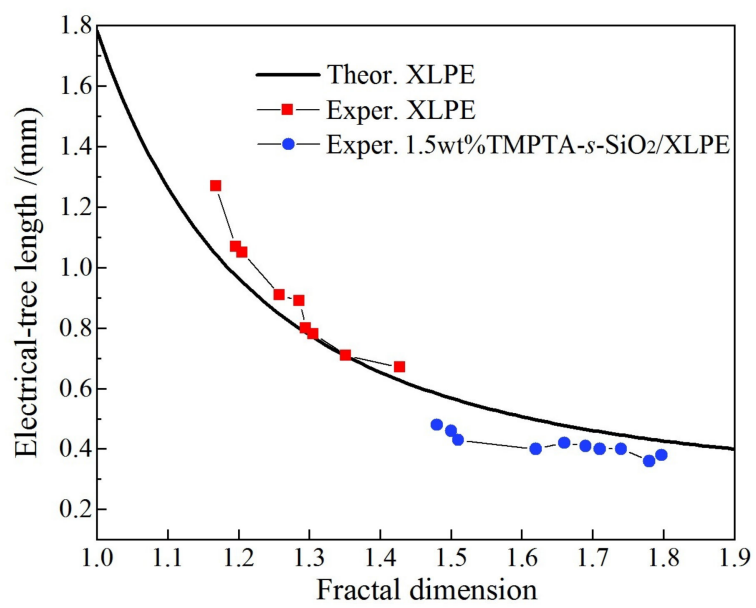

Figure 8. Growth length versus fractal dimension of electrical-trees in pure XLPE and $1.5 \mathrm{wt} \% \mathrm{TMPTA}$ $s-\mathrm{SiO}_{2} /$ XLPE nanocomposite.

\subsection{Charge Trapping Characteristics}

In order to investigate the deep-trapping mechanism in TMPTA-s-SiO ${ }_{2} / \mathrm{XLPE}$ nanocomposites that can enhance dielectric breakdown strength, energy level distributions of charge traps are evaluated from TSC results [23]. TSC testing temperatures from -30 to $175^{\circ} \mathrm{C}$ cover all of the required energies for thermal excitation of the charges captured in the deep traps introduced by nanosurface-grafting. The measured TSC temperature spectra and the derived trap densities as a function of trap energy level (trap level distributions) are shown in Figure 9. The general peaks arising in trap levels of $0.9-1.0 \mathrm{eV}$ originate from the intrinsic shallow traps introduced by the structural defects between XLPE lamellae.
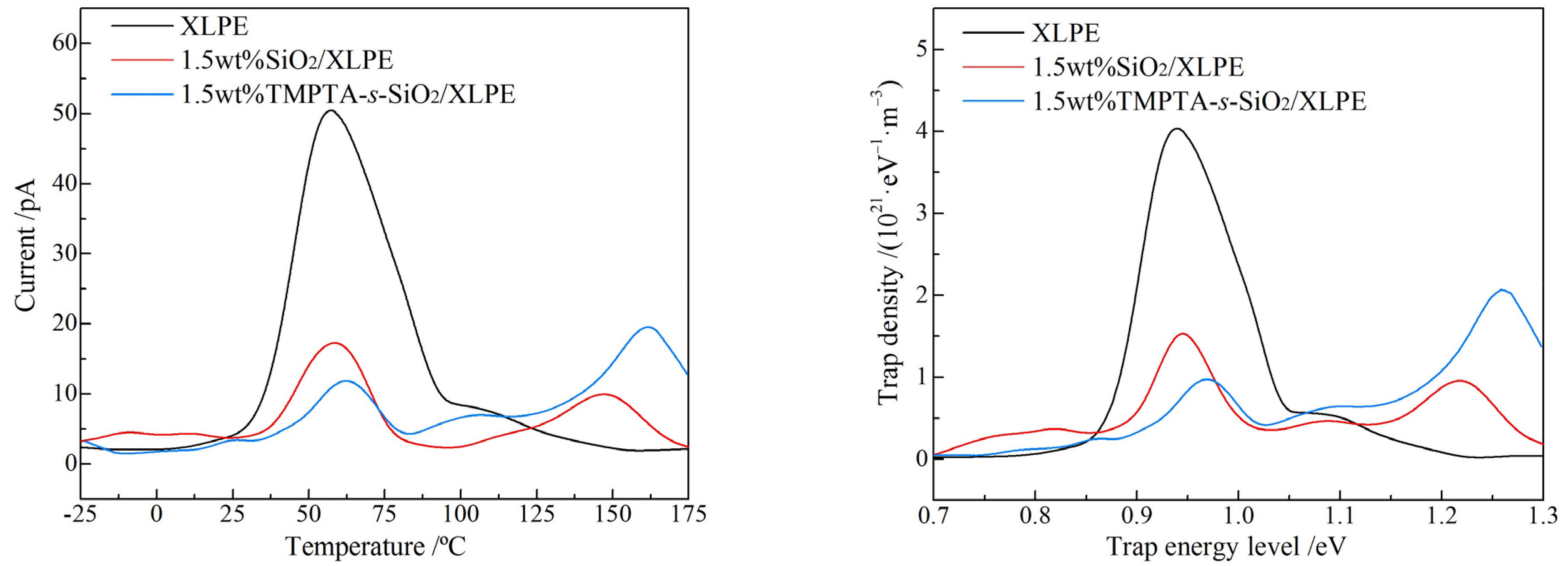

Figure 9. Thermally stimulated current (TSC) temperature spectra (left) and trap energy level distributions (right) of pure XLPE and the XLPE nanocomposites filled with TMPTA-s-SiO 2 and neat $\mathrm{SiO}_{2}$ nanoparticles, respectively.

Due to the deep traps introduced by TMPTA grafted onto surfaces of $\mathrm{SiO}_{2}$ nanofillers, a characteristic peak with lower amplitude and smaller integral area than XLPE intrinsic peak appears at $1.25 \mathrm{eV}$ for TMPTA-s- $\mathrm{SiO}_{2} / \mathrm{XLPE}$ nanocomposites, which is observably higher in peak intensity than that at $1.2 \mathrm{eV}$ for $\mathrm{SiO}_{2} / \mathrm{XLPE}$ nanocomposite which derives from discharging traps at nano- $\mathrm{SiO}_{2} / \mathrm{XLPE}$ interfaces. Because charge carriers prefer to be captured into deeper traps than shallower traps, no enough carriers have been trapped into intrinsic traps of XLPE matrix under high-voltage electric field in TMPTA-s-SiO 2 / XLPE nanocomposites. Therefore, the amplitude and integral area of trap distributions at $0.9-1.0 \mathrm{eV}$ are significantly lower than that of pure XLPE. It is proved by TSC tests that the surface functionalization of grafting TMPTA onto $\mathrm{SiO}_{2}$ nanoparticles has achieved deeper and 
higher density of charger traps in TMPTA-s-SiO $2 /$ XLPE nanocomposites than that in pure XLPE and $\mathrm{SiO}_{2} /$ XLPE nanocomposite, which will contribute to the improvement of electrical resistance.

\subsection{Dielectric Breakdown Strength}

Two-parameter Weibull statistics is utilized to analyze electrical breakdown fields under AC voltage, as shown in Figure 10. Scale parameter $E_{\mathrm{b}}$ characterizes the electrical breakdown field with a probability of $63.2 \%$, while shape parameter $\beta$ indicates the dispersivity of experimental data, as listed in Table 3.

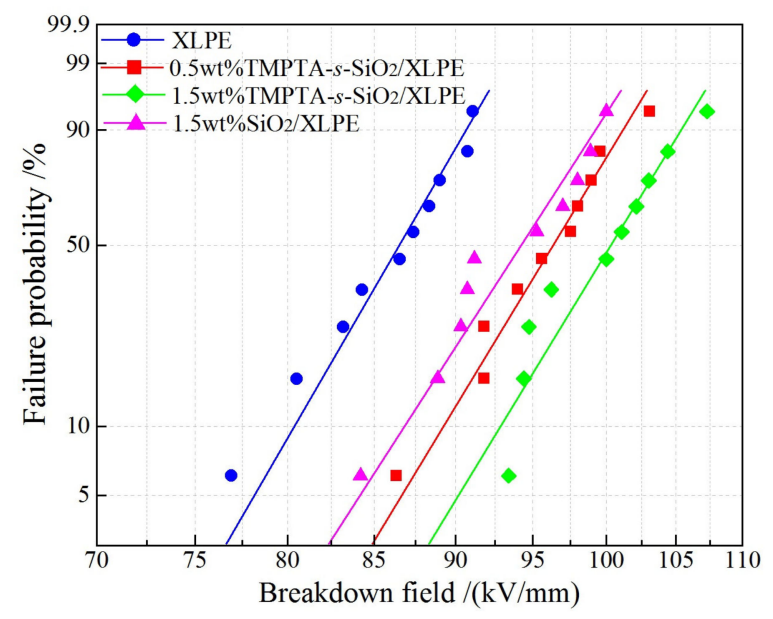

Figure 10. Electrical breakdown fields under AC voltage fitted with 2-parameter Weibull distribution for pure XLPE, TMPTA-s-SiO $2 /$ XLPE and $\mathrm{SiO}_{2} /$ XLPE nanocomposites.

Table 3. Characteristic breakdown field $E_{\mathrm{b}}$ at $63.2 \%$ probability and shape parameter $\beta$ obtained by fitting experimental results of electrical breakdown fields with 2-parameter Weibull distribution at $95 \%$ confidence interval.

\begin{tabular}{ccc}
\hline Materials & $\boldsymbol{E}_{\mathbf{b}} / \mathbf{( \mathbf { k V } / \mathbf { m m } )}$ & $\boldsymbol{\beta}$ \\
\hline XLPE & 87.74 & 25.74 \\
$0.5 \mathrm{wt} \% \mathrm{TMPTA}-\mathrm{s}-\mathrm{SiO}_{2} / \mathrm{XLPE}$ & 97.79 & 24.58 \\
$1.5 \mathrm{wt} \% \mathrm{TMPTA}-\mathrm{s}-\mathrm{SiO}_{2} / \mathrm{XLPE}$ & 101.83 & 24.42 \\
$1.5 \mathrm{wt} \% \mathrm{SiO}_{2} / \mathrm{XLPE}$ & 95.70 & 23.12 \\
\hline
\end{tabular}

DBS results in Weibull distributions prove that electrical breakdown performances of TMPTA-s-SiO ${ }_{2} /$ XLPE nanocomposites are better than that of pure XLPE and $\mathrm{SiO}_{2} / \mathrm{XLPE}$ nanocomposite. The relatively lower breakdown field of $1.5 \mathrm{wt} \% \mathrm{SiO}_{2} / \mathrm{XLPE}$ nanocomposite than that of TMPTA-s-SiO 2 /XLPE nanocomposites can be primarily attributed to the lack of "dielectric shielding" effects from nanosurface-modifications of grafting high polarizibility molecules to prevent electrical-trees developing directly towards ground electrode. The slightly increased breakdown field derives primiarily from the charge traps produced at interfaces between neat nanosilica and XLPE matrix [24], which however has been abated by the small specific surface area of the aggregated nanosilica caused by poor compatibility of $\mathrm{SiO}_{2}$ with polyethylene molecules. Meanwhile, XLPE nanocomposites filled with neat $\mathrm{SiO}_{2}$ nanoparticles will engender considerable particle agglomerations when filling content exceeds $1.5 \mathrm{wt} \%$. Furthermore, TMPTA molecules with polar-groups grafted on nanosilica surface will not be evaporated in crosslinking reactions, thus retaining a high density of effective polar-groups acting as deep traps to efficiently improve DBS under the charge trapping and scattering mechanisms [6,19]. It is thus suggested a reasonable correlation of DBS improvement with TMPTA surface-functionalization of nanosilica for XLPE-based nanodielectrics. These results support the charge-trapping mechanism and demonstrate 
that TMPTA is an effective graft candidate to improve the electrical resistance performances of polyethylene insulating materials.

\section{Conclusions}

In order to simultaneously ameliorate the crosslinking degree and electrical resistance of UV-XLPE insulating materials, chemical modification and nanodielectric technology are coordinately exploited by grafting auxiliary crosslink agent (TMPTA) onto nanosilica surface. FTIR and ${ }^{1} \mathrm{H}$-NMR characterizations demonstrate that TMPTA molecules have been successfully grafted onto nanosilica surfaces. TMPTA molecules being fixed on the surface of TMPTA-s-SiO $\mathrm{S}_{2}$ nanofillers disperse in polyethylene matrix acquire a high crosslinking efficiency without evaporation in crosslinking reaction, which could be further promoted by increasing the concentration of TMPTA-s-SiO 2 nanofillers. Furthermore, the larger sizes of neat $\mathrm{SiO}_{2}$-nanofillers in XLPE matrix is also a manifestation of inhibiting particle agglomerations by functional surface-modifications. TMPTA-s-SiO $2 / \mathrm{XLPE}$ nanocomposites show a higher tree inception voltage by about 19\% than pure XLPE and $\mathrm{SiO}_{2} /$ XLPE nanocomposite. The high polarizability layer of TMPTA covering $\mathrm{SiO}_{2}$ core can eliminate electric field in $\mathrm{SiO}_{2}$ core and highly increase electric field near outer surface of nanofillers, which can not be presented by neat $\mathrm{SiO}_{2}$ nanofillers. Due to the highly probable discharge near TMPTA-s-SiO${ }_{2}$ nanofiller surface, the electrical-trees are produced and propagating with a fast speed along the long circuitous pathways between TMPTA-s-SiO nanofillers, which accounts for the improvements in the fractal dimension and inception voltage of electrical-tree growth. In addition, polar-groups in TMPTA molecules grafted on nano-silica surfaces introduce effective deep traps in high density to further enhance DBS. TMPTA-s-SiO ${ }_{2} / \mathrm{XLPE}$ nanocomposites achieve comprehensive significant improvements in crosslinking degree, electrical-tree resistance and AC electrical breakdown field.

Author Contributions: Conceptualization, X.W.; Data curation and formal analysis, Y.-Q.Z.; Methodology, P.-L.Y.; Investigation and writing, W.-F.S.; Project administration, X.W. All authors have read and agreed to the published version of the manuscript.

Funding: This research was funded by the National Natural Science Foundation of China (grant no. 51337002).

Institutional Review Board Statement: Not applicable.

Informed Consent Statement: Not applicable.

Data Availability Statement: Experimental methods and results are available from the authors.

Conflicts of Interest: The authors declare no conflict of interest.

\section{References}

1. Ouyang, B.; Ming, H.; Deng, X. A review about development of HV XLPE cable materials and processes. Insul. Mater. 2016, 49, 1-6.

2. Ye, G.D.; Zhou, H.; Yang, J.W.; Zeng, Z.H.; Chen, Y.L. Photoinitiating behavior of macrophotoinitiator containing aminoalkylphenone group. J. Therm. Analy. Calor. 2006, 85, 771-777. [CrossRef]

3. Lu, Y.; Tang, J.; Zhao, H.; Hao, G.; Huang, B. Study of ventilation cooling for an irradiation box of low-voltage cable ultraviolet cross-linking. J. Harbin. Univ. Sci. Technol. 2013, 18, 45-50.

4. $\mathrm{Wu}, \mathrm{Q} . \mathrm{H}$.; Qu, B.J. Photoinitiating characteristics of benzophenone derivatives as new initiators in the photocrosslinking of polyethylene. Polym. Eng. Sci. 2001, 41, 1220-1226. [CrossRef]

5. Wu, Q.H.; Qu, B.J. Synthesis of di(4-hydroxyl benzophenone) sebacate and its usage as initiator in the photocrosslinking of polyethylene. J. Appl. Poly. Sci. 2002, 85, 1581-1586. [CrossRef]

6. Zhao, X.D.; Sun, W.F.; Zhao, H. Enhanced insulation performances of crosslinked polyethylene modified by chemically grafting chloroacetic acid allyl ester. Polymers 2019, 11, 592. [CrossRef]

7. Fu, Y.W.; Wang, X.; Wu, Q.H.; Zhao, H. Study of crosslinked and electrical characteristics for cable insulating material of new UV XLPE. Trans. Chin. Electrotech. Soc. 2018, 33, 178-186.

8. Chen, J.Q.; Zhao, H.; Zheng, H.F.; Chen, C.M.; Li, Y.; Sun, K. Research and design of electrodeless UV curing lamp with elliptic concentrator. Electr. Mach. Contr. 2017, 21, 109-113. 
9. Danikas, M.G.; Tanaka, T. Nanocomposites-a review of electrical treeing and breakdown. IEEE Electr. Insul. Mag. 2009, 25, 19-25. [CrossRef]

10. Tanaka, T. Dielectric nanocomposites with insulating properties. IEEE Trans. Dielectr. Electr. Insul. 2005, 12, 914-928. [CrossRef]

11. Kango, S.; Kalia, S.; Celli, A.; Njuguna, J.; Habibi, Y.; Kumar, R. Surface modification of inorganic nanoparticles for development of organic-inorganic nanocomposites-A review. Prog. Poly. Sci. 2013, 38, 1232-1261. [CrossRef]

12. Wemyss, A.M.; Bowen, C.; Plesse, C.; Vancaeyzeele, C.; Nguyen, G.T.M.; Vidal, F.; Wan, C. Dynamic crosslinked rubbers for a green future: A material perspective. Mater. Sci. Eng. R Rep. 2020, 141, 100561. [CrossRef]

13. Zhang, Y.; Khanbareh, H.; Roscow, J.; Pan, M.; Bowen, C.; Wan, C. Self-healing of materials under high electrical stress. Matter 2020, 3, 989-1008. [CrossRef]

14. Xu, J.W.; Pang, W.M.; Shi, W.F. Synthesis of UV-curable organic-inorganic hybrid urethane acrylates and properties of cured films. Thin Solid Films 2006, 514, 69-75. [CrossRef]

15. Sangermano, M.; Malucelli, G.; Amerio, E.; Priola, A.; Billi, E.; Rizza, G. Photopolymerization of epoxy coatings containing silica nanoparticles. Prog. Org. Coat. 2005, 54, 134-138. [CrossRef]

16. Medda, S.K.; Kundu, D.; De, G. Inorganic-organic hybrid coatings on polycarbonate.: Spectroscopic studies on the simultaneous polymerizations of methacrylate and silica networks. J. Non Cryst. Solids 2003, 318, 149-156. [CrossRef]

17. Crucho, C.I.C.; Baleizão, C.; Farinha, J.P.S. Functional group coverage and conversion quantification in nanostructured silica by ${ }^{1} \mathrm{H}$ NMR. Analyt. Chem. 2016, 89, 681-687. [CrossRef] [PubMed]

18. Sangermano, M.; Colucci, G.; Fragale, M.; Rizza, G. Hybrid organic-inorganic coatings based on thiol-ene systems. React. Funct. Poly. 2009, 69, 719-723. [CrossRef]

19. Zhao, X.D.; Zhao, H.; Sun, W.F. Significantly improved electrical properties of crosslinked polyethylene modified by UV-initiated grafting MAH. Polymers 2020, 12, 62. [CrossRef]

20. Qiu, P.; Chen, J.Q.; Sun, W.F.; Zhao, H. Improved DC dielectric performance of photon-initiated crosslinking polyethylene with TMPTMA auxiliary agent. Materials 2019, 12, 3540. [CrossRef] [PubMed]

21. Ding, H.Z.; Xing, X.S.; Zhu, H.S. A kinetic model of time-dependent dielectric breakdown for polymers. J. Phys. D Appl. Phys. 1994, 27, 591.

22. Chi, X.H.; Gao, J.G.; Zheng, J.; Zhang, X.H. The mechanism of electrical treeing propagation in polypropylene. Acta Phys. Sin. 2014, 63, 177701-177708.

23. Tian, F.; Bu, W.; Shi, L.; Yang, C.; Wang, Y.; Lei, Q. Theory of modified thermally stimulated current and direct determination of trap level distribution. J. Electrost. 2011, 69, 7-10. [CrossRef]

24. Roy, M.; Nelson, J.K.; MacCrone, R.K.; Schadler, L.S.; Reed, C.W.; Keefe, R. Polymer nanocomposite dielectrics-the role of the interface. IEEE Trans. Dielectr. Electr. Insul. 2005, 12, 629-643. [CrossRef] 